\title{
Reindução da Remissão Clínica com Adalimumabe após Interrupção do Tratamento: Uma Alternativa no Manejo da Doença de Crohn
}

\author{
Re-Induction of Clinical Remission with Adalimumab after Interruption of \\ Treatment: An Alternative Approach in Crohn's Disease Management
}

\author{
PAULO GUSTAVO KOTZE ${ }^{1}$; IDBLANCARVALHODE ALBUQUERQUE²; LORETE MARIA \\ DA SILVA KOTZE ${ }^{3}$; GALDINO JOSÉ SITONIO FORMIGA²
}

\begin{abstract}
1. Serviço de Coloproctologia do Hospital Universitário Cajuru (SeCoHUC-PUCPR); ${ }^{2}$ Serviço de Coloproctologia do Hospital Heliópolis - SP; ${ }^{3}$ Serviço de Gastroenterologia do Hospital Universitário Cajuru - PUCPR.
\end{abstract}

KOTZE PG; ALBUQUERQUE IC; KOTZE LMS; FORMIGA GJS. Reindução da Remissão Clínica com Adalimumabe após Interrupção do Tratamento: Uma Alternativa no Manejo da Doença de Crohn. Rev bras Coloproct, 2010;30(2): 135-140.

\begin{abstract}
RESUMO: Introdução: o Adalimumabe (ADA) é um anticorpo monoclonal totalmente humano, utilizado no tratamento da doença de Crohn (DC). Nos casos com necessidade de interrupção desta terapia, o resgate da resposta é controverso, e o papel da reindução da remissão com dose total de ataque pode ser uma alternativa nos casos graves da DC. Não há relatos desta forma de tratamento na literatura. $O$ objetivo deste estudo foi relatar a experiência de dois pacientes tratados com reindução da remissão com ADA, após sua interrupção. Método: análise retrospectiva de dois casos submetidos a reindução em meio a uma coorte de 24 pacientes em uso de ADA para DC. Resultados: são descritos dois casos de pacientes jovens, em tratamento com ADA subcutâneo, que tiveram necessidade de interrupção da terapia (um por abscesso perineal extenso e outro por dificuldade de acesso ao fornecimento da medicação). Ambos foram tratados pela reindução com dose total de ataque do ADA, com bons resultados, sem efeitos adversos. Conclusão: não há na literatura estudos controlados que comparem a reindução com outras formas de resgate da terapia após a parada de qualquer agente anti-TNF. Experiências isoladas mostram bons resultados com esta alternativa terapêutica em casos graves e selecionados.
\end{abstract}

Descritores: Doença de Crohn; Terapia biológica; Adalimumabe; Fator de necrose tumoral Alfa; Reindução.

\section{INTRODUÇÃO}

A terapia biológica é um dos tratamentos mais utilizados no manejo terapêutico atual da doença de Crohn (DC). Desde a utilização do Infliximabe (IFX), primeiro agente biológico liberado para o tratamento da DC no final dos anos 90, consideráveis avanços foram atingidos ${ }^{1}$. Após mais de 10 anos de prática clínica com o IFX ${ }^{2}$, em 2007 foi liberado o uso do Adalimumabe (ADA) para a terapia da DC ${ }^{3,4}$.

O ADA é um anticorpo monoclonal totalmente humano, de uso subcutâneo, inibidor do fator de necrose tumoral alfa (TNF-alfa) utilizado no tratamento da DC moderada a grave ${ }^{3}$. Sua eficácia na indução e manutenção da remissão clínica foi documentada na literatura através de alguns estudos clínicos controlados ${ }^{3,4}$. Há trabalhos que demonstraram maior eficácia na utilização contínua do tratamento com medicamentos anti-TNF em relação ao seu uso episódico ${ }^{5}$. Experiências de grandes centros mostram eficácia clínica sustentada por muitos anos, tanto com o IFX ${ }^{6}$ como com o ADA ${ }^{7}$. Eventualmente, tem-se a necessidade de interrupção do uso destas drogas por causas variadas. As alternativas de resgate da terapia, nestes ca-

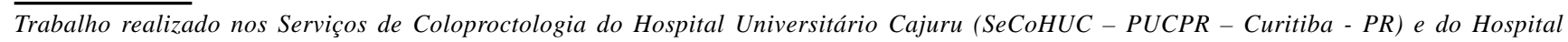
Heliópolis - São Paulo - SP - Brasil. 
Paulo Gustavo Kotze e Cols.

sos, são controversas, e diversas opções tem sido descritas na literatura, como o aumento de doses e a diminuição dos intervalos de medicação, por exemplo ${ }^{8}$.

Diferentemente do manejo da artrite reumatóide (AR), na DC há a necessidade de uma dose de ataque para melhores resultados clínicos, de acordo com os estudos controlados, tanto com o IFX como com o ADA ${ }^{1,3}$. Uma alternativa nas situações de interrupção do tratamento (por efeitos adversos, perda de resposta, ou outros fatores) seria a reindução da terapia com dose total de ataque, principalmente em casos graves da doença. Dados sobre esta estratégia são escassos na literatura.

O objetivo deste trabalho é apresentar a experiência de dois casos, em uma coorte de pacientes portadores de DC que utilizam o ADA, que foram manuseados com a reindução da droga com dose total, após a suspensão do tratamento por motivos variados. Através destas descrições, pretende-se discutir a eficácia desta alternativa, já que não há relatos na literatura sobre o tema.

\section{MÉTODO}

Trata-se de um estudo retrospectivo de dois casos, em uma coorte de portadores de DC que utilizam o ADA como forma principal de tratamento. A coleta de dados foi feita através do acesso aos registros médicos (prontuários) dos pacientes, registrando-se várias informações, com preenchimento de protocolo específico de portadores de DC em uso de terapia biológica. Todos os dados foram coletados por um pesquisador, sendo compilados para posteriores análises e avaliações.

\section{RESULTADOS}

Em uma coorte de 24 portadores de DC com utilização contínua de ADA, foram incluídos nesta análise dois pacientes que tiveram a utilização da medicação interrompida, por diferentes motivos. Os dois casos são apresentados a seguir.

\section{Caso 1}

Paciente masculino, 33 anos, portador de DC pancolônica e perianal há 12 anos. Após perda de resposta ao tratamento com IFX, iniciou há um ano terapia com ADA com boa resposta. Desenvolveu extenso abscesso perianal, na vigência da medicação (figura 1). Foi submetido a exame proctológico sob anestesia com drenagem do abscesso, locação de sedenhos, terapia antimicrobiana e suspensão do $\mathrm{ADA}$, devido a sepse pélvica (figuras 2 e 3 ). Após quatro semanas da

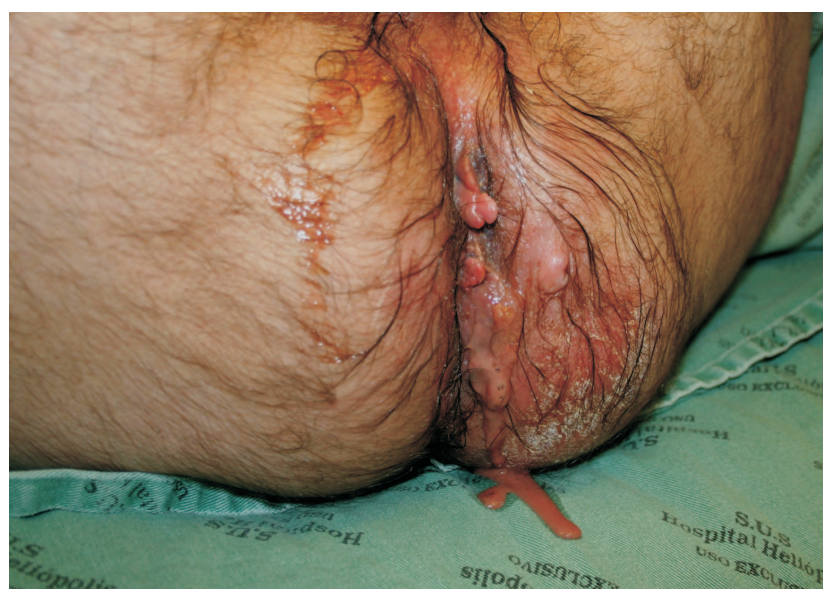

Figura 1 - Extenso abscesso perianal durante o uso do ADA (caso 1).



Figura 2 - Detalhe do ato cirúrgico de drenagem do abscesso perianal extenso, com identificação das lojas e cateterização dos trajetos (caso 1).

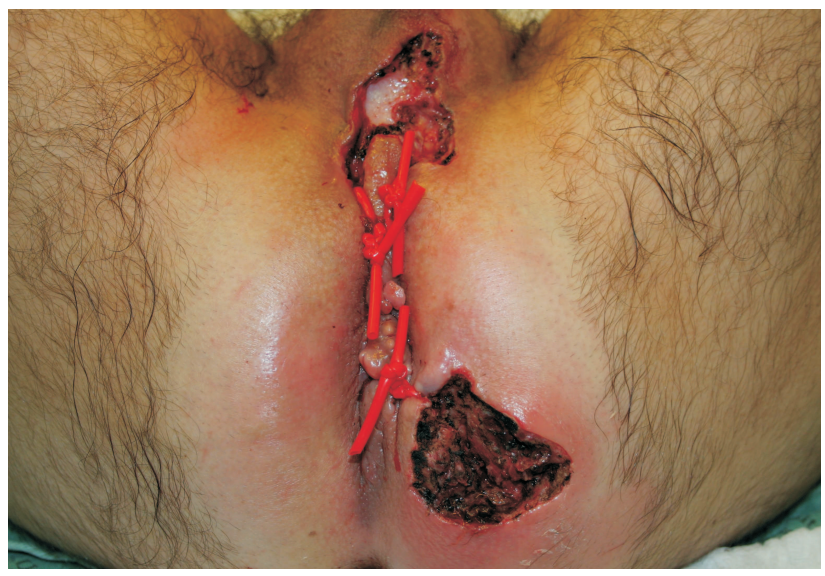

Figura 3 - Aspecto pós-operatório imediato após drenagem das lojas de abscesso e locação de sedenhos nos trajetos fistulosos (caso 1). 
suspensão do medicamento, apresentou grave crise de agudização, com aumento das provas de atividade inflamatória e diarréia. Realizou reindução com ADA 160 mg na semana $0,80 \mathrm{mg}$ na semana 2 e $40 \mathrm{mg}$ a cada 2 semanas, seguida de curetagens das fístulas e retirada dos sedenhos, com excelente resposta clínica e tolerabilidade. Após cinco meses da reindução da remissão com dose de ataque, apresentou melhora significativa da região perianal e cicatrização das feridas cirúrgicas, encontrando-se assintomático e em tratamento de manutenção até os dias atuais (figura 4).

\section{Caso 2}

Paciente masculino, 29 anos, portador de DC há 23 anos. Há 13 anos submetido a colectomia total com ileostomia terminal, por pancolite de Crohn e intratabilidade clínica. Ao exame físico apresentava fístulas peri-ileostômicas com extenso processo inflamatório na pele peri-estomal (figuras 5 e 6). Queixava-se de dificuldades no manuseio de placas e bolsas na ileostomia, com dermatite secundária. Realizou 3 infusões de IFX, com reação alérgica infusional na terceira aplicação. Iniciou tratamento com ADA subcutâneo, mantido por três meses, com melhora dos sintomas. Interrompeu o tratamento por dificuldade de acesso ao medicamento, tanto pelo sistema público como pelo sistema privado de saúde. Houve reativação da doença, com grave crise de agudização após 45 dias, com significativo aumento da secreção das fístulas ao redor da ileostomia. Após regularização do fornecimento do ADA para o paciente, devido à gravidade do quadro, foi realizada a reindução da remissão clínica com 160 mg na semana $0,80 \mathrm{mg}$ na semana 2 e $40 \mathrm{mg}$ a cada 2 semanas, com bom resultado. Foi submetido à cirurgia para mudança de sitio da ileostomia e ressecção do segmento distal da ileostomia prévia, por acometimento extenso da DC na pele ao redor do estoma (figuras 7 e 8). Após um ano de tratamento, o paciente encontrava-se assintomático, em esquema de manutenção, com cicatrização total das feridas (figura 9).

\section{DISCUSSÃO}

A eficácia e os significativos índices de sucesso no tratamento de portadores de DC com a terapia biológica são amplamente documentados na literatura 1,3,4,9. Dados demonstrando sua segurança, com índices de efeitos adversos semelhantes aos de pacientes em tratamento convencional (baseado somente com

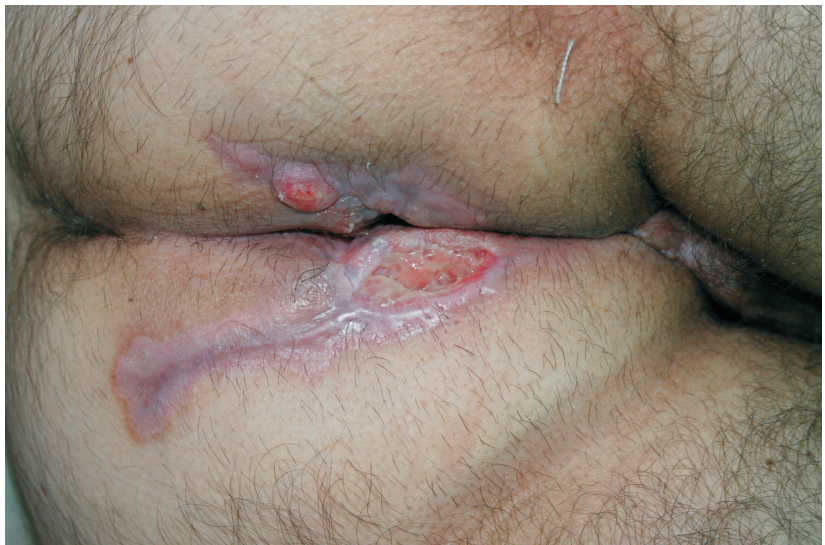

Figura 4 - Aspecto da cicatrização após reindução do tratamento com ADA em dose de ataque. Fechamento da ferida e tecido de granulação (caso 1).

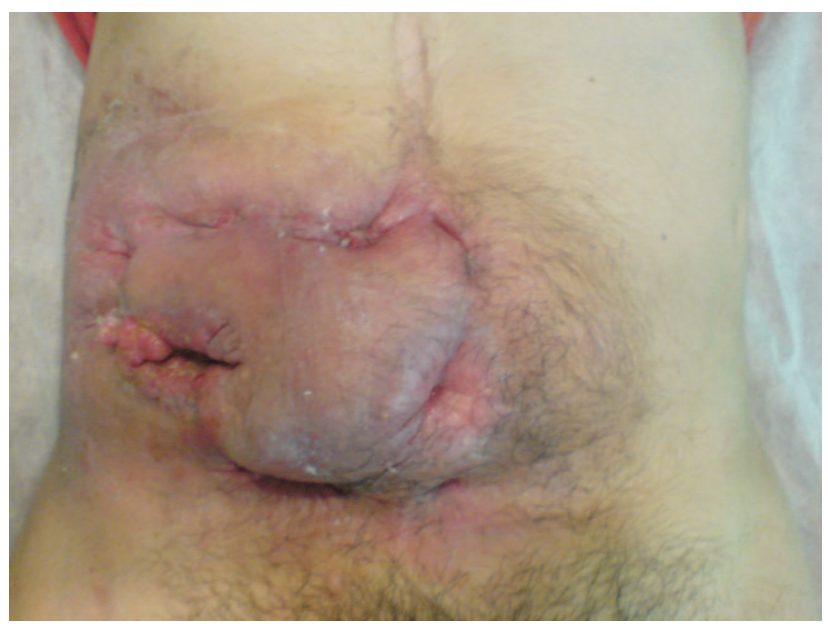

Figura 5 - Múltiplas fístulas para-ileostômicas na fossa ilíaca direita, após colectomia total e ileostomia terminal há 13 anos (caso 2).

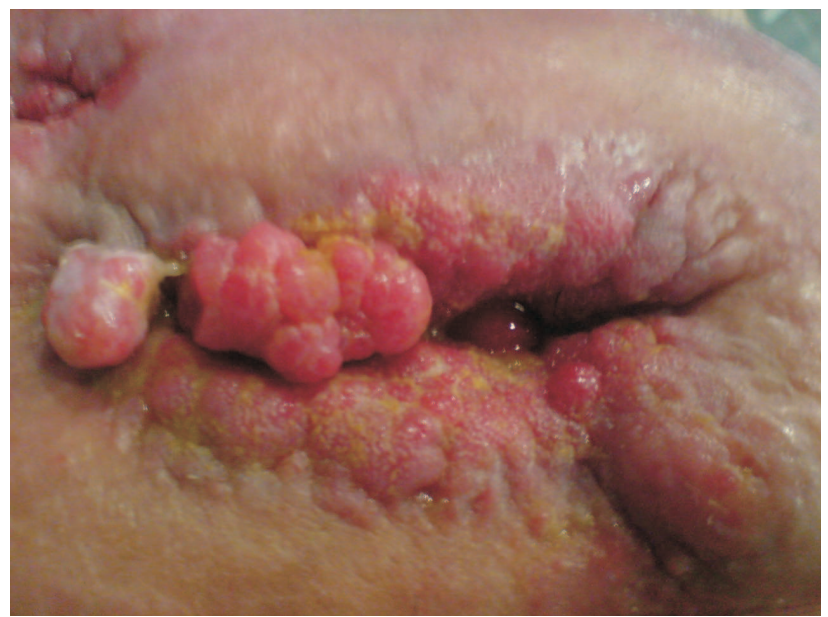

Figura 6 - Detalhes do aspecto granulomatoso da DC no local da ileostomia e na pele peri-estomal (caso 2). 


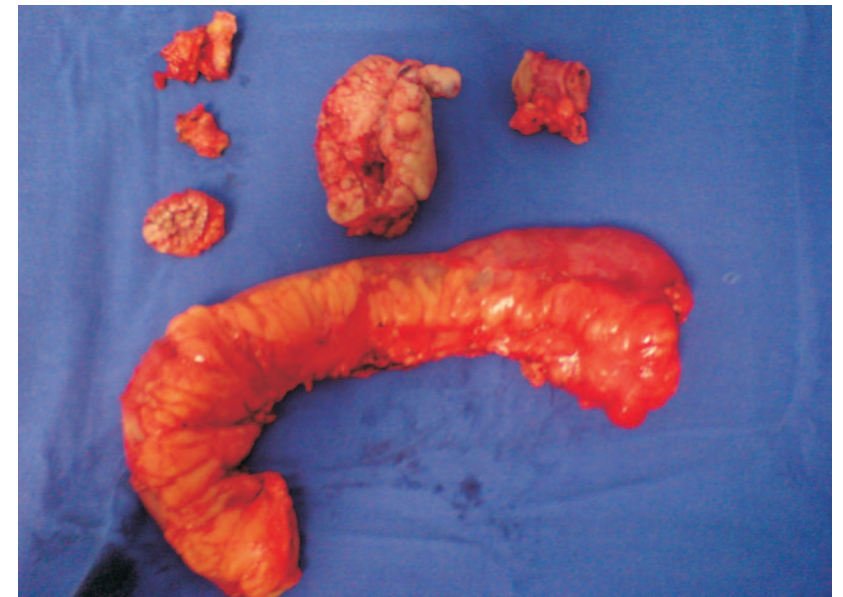

Figura 7-Aspecto das peças da ressecção cirúrgica do ileo terminal e da ileostomia prévia, com comprovação histológica de DC na pele (caso 2).

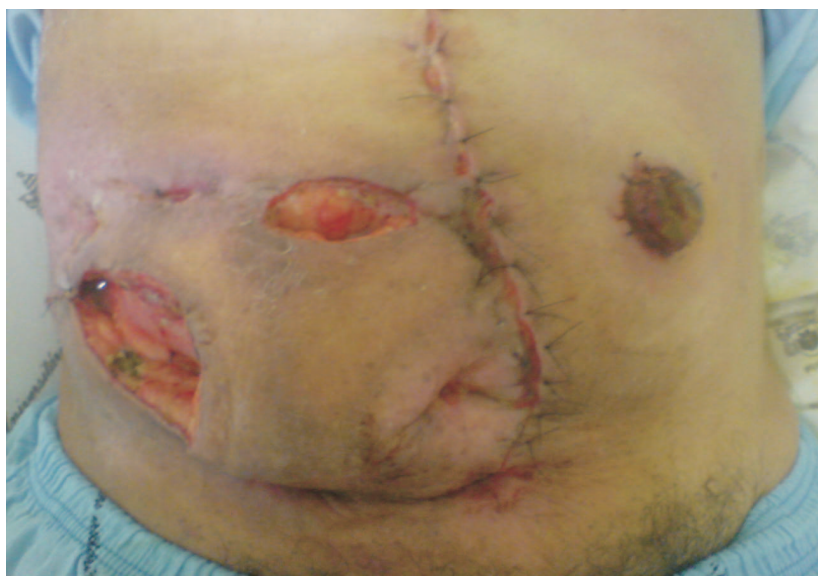

Figura 8 - Aspecto do pós-operatório imediato, com ileostomia locada na fossa ilíaca esquerda, e curetagem dos trajetos das fístulas prévias (caso 2).

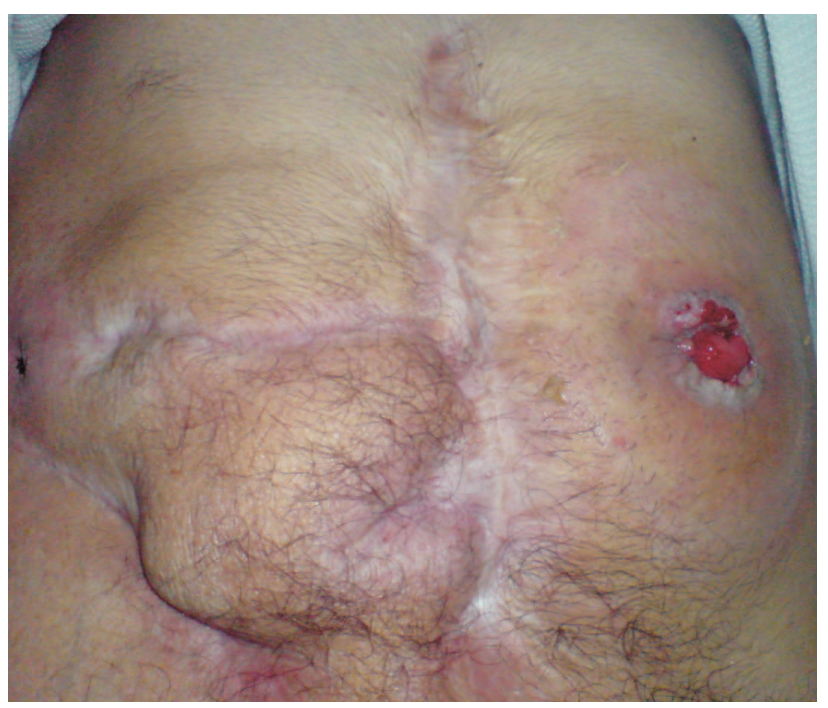

Figura 9 - Cicatrização total das feridas após um ano da reindução com ADA (caso 2). imunossupressores) foram exaustivamente estudados ${ }^{2,5,10}$. Estes aspectos são claros tanto na utilização do IFX quanto do ADA no manuseio desta difícil entidade.

Entretanto, mesmo com a crescente utilização destes medicamentos na prática clínica atual, há considerável parcela de pacientes que necessitam de interrupção no seu tratamento. As razões para esta interrupção são múltiplas. Efeitos adversos como infecções oportunistas, desmielinização do sistema nervoso central e periférico, além de reações alérgicas secundárias às aplicações dos medicamentos podem ocorrer com freqüência variável ${ }^{7}$. Há contraindicação absoluta para o uso de medicamentos biológicos na vigência de qualquer infecção em tratamento, o que comprova a necessidade da interrupção dos mesmos. Além disso, no nosso meio, pelo alto custo isolado destas drogas, a dificuldade dos pacientes em se conseguir os medicamentos ainda são significativas, e frequentemente, o não-fornecimento das drogas pode ocorrer, tanto no cenário público quanto privado de saúde ${ }^{11}$. Nestas situações, os pacientes podem ser prejudicados, e crises de agudização podem ocorrer, pela interrupção dos medicamentos. Nos casos descritos, houve necessidade de suspensão do tratamento por sepse pélvica (caso 1) e por dificuldade de acesso contínuo à droga (caso 2 ), e os dois pacientes apresentaram crises de agudização após a suspensão do agente anti-TNF.

A perda de resposta aos medicamentos é outro aspecto que deve ser relevado. Os estudos de uso contínuo com IFX e ADA mostram que a diminuição e posterior ausência de resposta a estes fármacos ocorre com certa freqüência ${ }^{1,4}$. Nestas situações, estratégias de resgate da terapia foram e continuam sendo estudadas. Alguns trabalhos sugerem que a redução do intervalo das medicações (de 8 para 6 semanas no IFX ou de 2 para 1 semana no ADA) pode causar melhora dos sintomas e até remissão ${ }^{4,8}$. No caso do IFX, o aumento da dose de $5 \mathrm{mg} / \mathrm{kg}$ para $10 \mathrm{mg} / \mathrm{kg}$ foi bem avaliado nos estudos controlados e em outros trabalhos, com recuperação da resposta clínica ${ }^{1,8,9}$. Entretanto, a conduta com estes pacientes é controversa. Se uma redução à dose original deve ser preconizada, e quando seria o melhor momento para isto, permanece obscuro na revisão bibliográfica realizada.

Não há na literatura revisada qualquer relato de reindução da remissão clínica com novas doses de ataque com IFX ou ADA na DC. Informalmente, sabese de alguns casos onde a reindução foi realizada, po- 
rém, nada sobre esta alternativa encontra-se publicado. O conhecimento sobre a terapia biológica encontra-se bem avançado na reumatologia, pela grande utilização destes medicamentos principalmente no manuseio da artrite reumatóide (AR). Entretanto, como nesta afecção não há necessidade de dose de ataque para remissão clínica, não há qualquer estudo ou relato sobre reindução de tratamento nesta especialidade. Há um caso relatado, em meio a uma série, com reindução da remissão com IFX no manuseio de episclerite ${ }^{12}$. Esta alternativa de reindução está descrita em um trabalho não publicado sobre o certolizumabe pegol, agente biológico liberado para DC nos Estados Unidos e Suíça, entre outros países ${ }^{13}$. Salienta-se que esta descrição foi em pacientes com perda de resposta, e não após a interrupção da droga. Aguarda-se a publicação de estudos demonstrando esta possibilidade.

Devido a esta escassez de dados, obviamente não há qualquer estudo que comprove que a reindução da remissão com dose de ataque é superior ou não às estratégias de resgate de tratamento pela redução do intervalo ou aumento de doses das medicações. Não se sabe igualmente se a reindução pode trazer maiores índices de efeitos adversos em relação às outras duas estratégias. Claramente o objetivo dos presentes relatos não é o de se recomendar esta estratégia para qualquer paciente, pois melhores avaliações e comparações em números maiores de casos são necessárias. Nos pacientes relatados não houve qualquer efeito adverso e a resposta clínica foi muito boa, com a remissão sendo atingida nos dois casos.

Conclui-se que a estratégia da reindução da remissão com dose total de ataque é uma alternativa viável em portadores de DC que necessitem de interrupção do tratamento e posterior reutilização das drogas. Os casos relatados ocorreram com o ADA, porém, estes conceitos podem ser aplicados também para o uso do IFX ou outros agentes anti-TNF. O bom senso no momento do tratamento deve prevalecer, e casos graves e selecionados podem ser tratados com esta atraente alternativa. Estudos controlados e comparativos são necessários para se definir qual a melhor forma de resgate do tratamento biológico após sua interrupção.

\begin{abstract}
Introduction: Adalimumab (ADA) is a fully-human anti-TNF-alpha antibody approved for the management of Crohn's disease (CD). In situations in which interruption of the treatment is mandatory, rescue therapy is controversial. Reinduction of remission with full dose may be an alternative in severe CD cases. There are no descriptions of this alternative in the literature. The aim of this study was to describe the experience with two patients treated with reinduction of remission with ADA, after interruption of treatment. Method: retrospective analysis of two patients in a cohort of 24 CD patients treated with ADA, with case reports. Results: description of two young patients with fistulizing CD, with the need of interruption of ADA therapy due to different reasons (perianal abscess and difficulties in receiving the medication). Both were treated with reinduction of remission with full dose ADA regimen $(160 / 80 \mathrm{mg})$, with good results and no adverse events. Conclusions: there are no studies in the literature comparing reinduction of remission with other possibilities of rescue therapy after interruption of treatment with any anti-TNF agent. Small case series like this, show good results with this alternative in selected and severe cases of CD.
\end{abstract}

Key words: Crohn’s disease; Biological Therapy; Adalimumab; Tumor-necrosis-factor Alpha; Reinduction.

\section{REFERÊNCIAS}

1. Hanauer SB, Feagan BG, Lichtenstein GR, Mayer LF, Schreiber $\mathrm{S}$, Colombel JF, et al. Maintenance infliximab for Crohn's disease: the ACCENT I randomised trial. Lancet 2002;359:1541-1549.

2. Cornillie F. Ten years of infliximab (Remicade $\AA$ ) in clinical practice: The story from bench to bedside. Eur Journal of Pharmacol 2009;623:S1-S4.

3. Hanauer SB, Sandborn WJ, Rutgeerts P, Fedorak RN, Lukas $\mathrm{N}$, Macintosh D, et al. Human anti-tumor necrosis factor monoclonal antibody (adalimumab) in Crohn's disease: the CLASSIC-I trial. Gastroenterology 2006;130(2):323-333.
4. Colombel JF, Sandborn WJ, Rutgeerts P, Enns R, Hanauer $\mathrm{SB}$, Panaccione R, et al. Adalimumab for maintenance of clinical response and remission in patients with Crohn's disease: the CHARM trial. Gastroenterology 2007;132(10):52-65.

5. Rutgeerts P, Van Assche G, Vermeire S. Review article: infliximab therapy for inflammatory bowel disease - seven years on. Aliment Pharmacol Ther 2006;23:451-463.

6. Schnitzler F, Fidder H, Ferrante M, Noman M, Arijs I, Van Assche G, Hoffman I, Van Steen K, Vermeire S, Rutgeerts P. Long-term outcome of treatment with infliximab in 614 patients with Crohn's disease: results from a single-centre cohort. Gut 2009;58(4):492-500. 
7. Colombel JF, Schwartz DA, Sandborn WJ, Kamm MA, D'Haens G, Rutgeerts P, Enns R, Panaccione R, Schreiber S, Li J, Kent JD, Lomax KG, Pollack PF. Adalimumab for the treatment of fistulas in patients with Crohn's disease. Gut 2009;58(7):940-948.

8. Regueiro M, Slemanowski B, Kip KE, Plevy S. Infliximab dose intensification in Crohn's disease. Inflamm Bowel Dis 2007;13:1093-1099.

9. Sands BE, Anderson FH, Bernstein CN, Chey WY, Feagan BG, Fedorak RN, et al. Infliximab maintenance therapy for fistulizing Crohn's Disease. N Engl J Med 2004;350:876-885.

10. Colombel JF. Efficacy and safety of adalimumab for the treatment of Crohn's disease in adults. Expert Rev Gastroenterol Hepatol 2008;2(2):163-176.

11. Kotze PG; Albuquerque IC; Moraes AC; Vieira A; Souza F. Análise de Custo-Minimização entre o Infliximabe (IFX) e o
Adalimumabe (ADA) no Tratamento da Doença de Crohn (DC). Rev bras Coloproct, 2009;29(2): 158-168.

12. Sen HN, Sangave A, Hammel K, Levy-Clarke G, Nussenblatt RB. Infliximab for the treatment of active scleritis. Can J Ophthalmol 2009;44(3):e9-e12.

13. Lichtenstein G, Mitchev K, D'Haens G. Re-indutcion with certolizumab pegol following disease exacerbation during maintenance therapy is effective to regain response and remission. J Crohn's and Colitis 2008;2:12 (P022).

\section{Endereço para correspondência:}

\section{PAULO GUSTAVO KOTZE}

Rua Jaime Balão, 975 - ap. 106 - Hugo Lange

80040-340 - Curitiba - PR

Fone/FAX: (41) 3022-5500

E-mail:pgkotze@hotmail.com 